\title{
The Emitting Envelope of Pleione between 1987 and $1998^{1}$
}

\author{
D. Ballereau, J. Chauville
}

Observatoire de Paris-Meudon - DASGAL/UMR 8633 du CNRS, F-92195 Meudon Principal Cedex, France

\section{J. Zorec}

Institut d'Astrophysique de Paris - CNRS - $98^{\mathrm{bis}}$ bd. Arago, F-75014 Paris, France

\section{N. Morrell}

La Plata Observatory, Paseo del Bosque s/n - 1900 La Plata, Argentina

\begin{abstract}
.
We analyze a series of spectroscopic observations of the $\mathrm{H} \beta$ and $\mathrm{Fe}$ II $\lambda 4924 \AA$ lines of the Be-shell star Pleione (HD23862), which entered a new Be phase since 1988. Their evolution show that the $\mathrm{H} \beta$-emitting envelope expands regularly from 1987 to $1996\left(r / r_{*}=8.6\right.$ to 25.0$)$, then diminishes slightly in 1997-98 $\left(r / r_{*}=20.0\right)$. The Fe II-emitting envelope expands regularly between 1987 and $1998\left(r / r_{*}=6.7\right.$ to 13.4$)$, but remains always inside the $\mathrm{H} \beta$-one. The evolution of the emitting envelope of Pleione is similar to the one observed between 1954 and 1972, and this one should decrease regularly in the next years, if the former scenario is repeated.
\end{abstract}

\section{Introduction}

The Be-shell star Pleione (28 Tau, HD23862, B8Vpe, $v \sin i=320 \mathrm{kms}^{-1}$ ) is one of the most known active Be star. Since 1888, it displayed several B, Be and Be-shell phases; the present one is a Be phase (1988-1998). From the general behaviour of the emission on the optical spectrum, it is admitted that Pleione has a period of activity of $34 \mathrm{yr}$. The duplicity of Pleione was established by Katahira et al. (1996), with a 218.0 d-period.

We present here a new series of 17 spectroscopic observations of Pleione obtained over 11 yr (1987-1998), which concern the $\mathrm{H} \beta$ and the Fe II $\lambda 4924 \AA$ line profiles observed simultaneously, and permit to follow the evolution of the emitting circumstellar envelope (CE). These observations extend those published by Ballereau et al. (1988) since 1978 to the end of the shell episode, around 1987. Two of these profiles were kindly provided by Dr. A. Slettebak, and their spectral resolutions were between 11500 and 18000 . The Table 1 gives the main

\footnotetext{
${ }^{1}$ Data obtained at CASLEO (Argentina) and Haute-Provence (France) Observatories
} 


\begin{tabular}{|l|rllllr|llll|}
\hline & $\mathrm{H} \beta$ & & & & & & Fe II & & \\
\hline year & $\Delta \mathrm{E}$ & $\Delta \mathrm{e}$ & $\mathrm{V} / \mathrm{R}$ & $\mathrm{RVe}$ & $-W \mathrm{e}$ & $r / r_{*}$ & $\Delta \mathrm{e}$ & $-W \mathrm{e}$ & $\mathrm{RVe}$ & $r / r_{*}$ \\
\hline 1987 & 592 & 218 & 0.80 & -16 & 2.38 & 8.6 & & & & \\
1988 & 596 & 210 & 0.79 & -16 & 2.30 & 9.3 & & & & \\
1989 & 587 & 200 & 0.83 & -22 & & 10.2 & & & & \\
1994 & 638 & 151 & 0.77 & -27 & & 18.0 & 248 & 0.21 & -96 & 6.7 \\
1995 & 678 & 142 & 0.79 & 7 & 3.54 & 20.3 & 221 & 0.21 & -25 & 8.4 \\
1996 & 668 & 128 & 0.81 & 12 & 3.46 & 25.0 & 200 & 0.30 & -22 & 10.2 \\
1997 & 645 & 146 & 0.86 & 57 & 3.05 & 19.2 & 195 & 0.32 & -16 & 10.8 \\
1998 & 645 & 140 & 0.93 & 69 & 3.58 & 20.9 & 175 & 0.52 & -6 & 13.4 \\
\hline
\end{tabular}

Table 1. Some measurements and calculations from the $\mathrm{H} \beta$ and $\mathrm{Fe}$ II $\lambda 4924 \AA$ line profiles. The widths and RVs are in $\mathrm{kms}^{-1}$ and the equivalent widths in $\AA$.

No. of spectra averaged each year are (resp.): $12,13,2,1,4,4,1,1$ $\mathrm{H} \beta$ : $\Delta \mathrm{E}$ : total width of the emission line, measured at the bottom of the emission wings; $\Delta \mathrm{e}$ : emission peak separation;

V/R: ratio of the violet to the red emission peak;

RVe: radial velocity of the whole emission, measured as for $\Delta \mathrm{E}$;

$-W e$ : equivalent width of the emission, measured on the profile corrected from the photospheric profile; $r / r_{*}$ : outer radius of the emitting region, in stellar radii.

Fe II: $\mathrm{RVe}=$ radial velocity of the whole emission, measured at the middle of the two emission peaks

data of these profiles, averaged per year. Figures 1 and 2 show the intensity line profiles of these two lines, at three particular epochs.

\section{Evolution of the $H \beta$ and Fe II $\lambda 4924 \AA$ line profiles (1987-1998)}

Let us recall the previous results from the 1978-1987 epoch :

1. The maximum strength of the shell lines occurs in 1981 ;

2. The RVs of shell lines show low amplitude variations until 1984;

3. The shell components of $\mathrm{H} \beta$ and metallic ions begin to vanish in 1984-85; their RVs become more and more negative, while the Balmer lines show a more and more negative progression;

4. Emission on $\mathrm{H} \beta$ increases gradually from 1984 , and its $V / R$ ratio becomes progressively less than unity;

5. All these phenomena can be understood in terms of a progressive increase of the extent of the circumstellar envelope.

The evolution of the $\mathrm{H} \beta$ line profile in 1987-98 (Table 1) is the following:

1. the central shell line, present in $1987-88(V / R=0.79)$ vanishes in 1994;

2. the 1994-95-96 profiles show a prominent red emission and a tiny violet emission and the V/R ratio increases slowly from 0.77 to 0.83 ;

3. the 1997 and 1998 profiles show an increase of the violet emission component and the $V / R$ ratio is 0.86 , then 0.93 ;

4. the equivalent width of the emission component, normalized to the photospheric profile, increases from $W=-2.34 \AA(1987-88)$ to a mean value of $W=$ 


\section{$-3.41 \AA(1995-98)$;}

5. the RV of the whole emission, measured at the bottom of the wings, undergoes a constant increase from $-16 \mathrm{kms}^{-1}(1987-88)$ to $+69 \mathrm{kms}^{-1}(1998)$;

6 . the total width of the emission, measured at the bottom of the wings, increases from $546 \mathrm{kms}^{-1}(1987-88)$ to $678 \mathrm{kms}^{-1}$ (1995), then diminishes to 645 $\mathrm{kms}^{-1}$ (until 1998);

7. the emission peak separation decreases from $220 \mathrm{kms}^{-1}$ (1987) to $128 \mathrm{kms}^{-1}$ (1996), then increases up to $143 \mathrm{kms}^{-1}(1997-98)$.
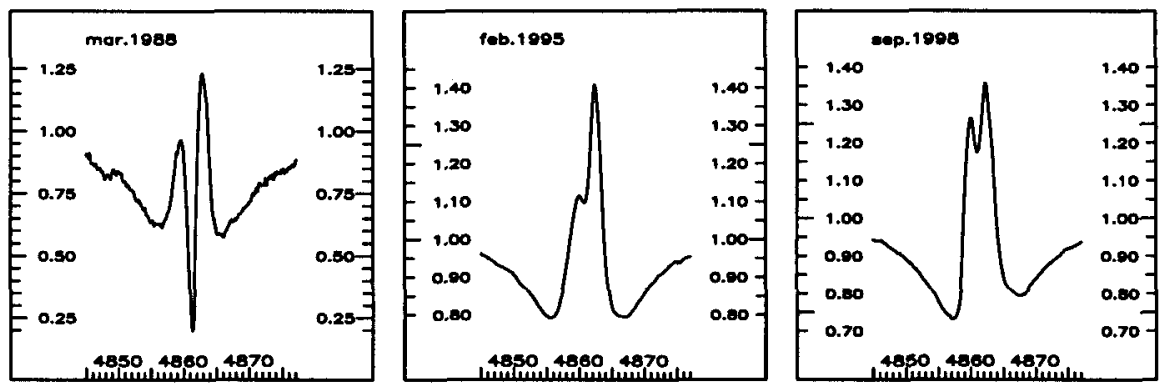

Figure 1. The $\mathrm{H} \beta$ line profile of Pleione (HD23862) at three selected epochs between 1987 and 1998

For the Fe II $\lambda 4924 \AA$ line profile, the evolution, in the same period of time can be summarized as follows:

1. in 1987-88, it displays a P-Cyg profile, with a strong shell component ;

2. from 1994, the shell component disappears and a violet emission component begins to appear $(V / R \ll 1)$; this last increases until 1997 , when the $V / R$ ratio begins to reach 1 ;

3. from 1994, a third and small emission component often appears on the red wing of the red emission component (around $\mathrm{RV}=+300 \mathrm{kms}^{-1}$ ) ;

4. the RV of the whole emission line, measured at the middle of the emission peaks, increases from $-96 \mathrm{kms}^{-1}$ (1994) to $-6 \mathrm{kms}^{-1}(1998)$;

5. the emission peak separation diminishes from $248 \mathrm{kms}^{-1}$ (1994) to $175 \mathrm{kms}^{-1}$ (1998);

6. the equivalent width of the emission peaks increases from $W=-0.21 \AA$ (1994) to $W=-0.52 \AA$ (1998).

\section{Evolution of the circumstellar envelope from 1987 to 1998}

Huang's relation (1972) gives an approximation to the outer radius of the emitting region in the $\mathrm{CE}$ :

$$
r / r_{*}=(2 v \sin i / \Delta \mathrm{e})^{1 / j}(j=1 / 2, \text { Keplerian case })
$$

where $v \sin i$ is the projected rotational velocity of the central star and $\Delta \mathrm{e}$ is the emission peak separation. The radius estimated using the $\mathrm{H} \beta$ line, increases continuously from $r / r_{*}=8.6(1987)$ to $25.0(1996)$, and then diminishes to a mean value of 20.0 in $1997-98$. 

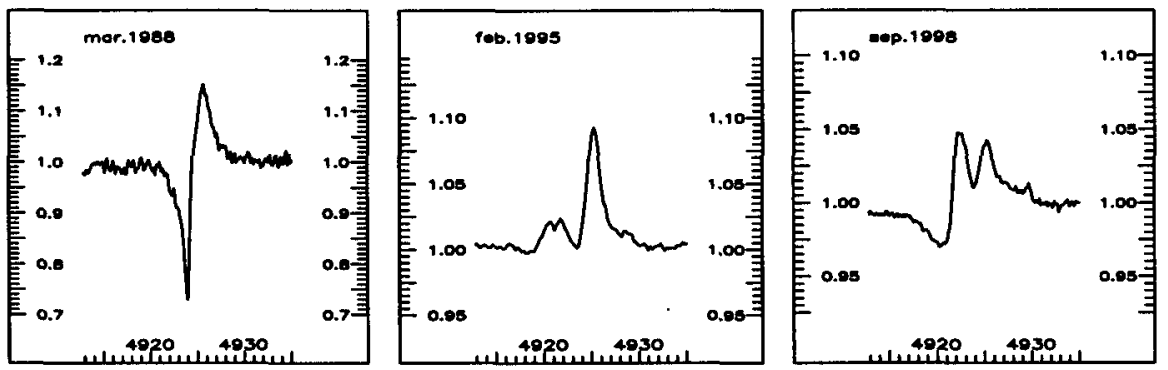

Figure 2. The Fe II $\lambda 4924 \AA$ line profile of Pleione (HD23862) at three selected epochs between 1987 and 1998

For the Fe II emitting region, the radius increases continuously from $r / r_{*}$ $=6.7$ in 1994 to 13.4 in 1998 .

\section{Conclusions}

Between 1978 and 1987, the hydrogen emitting region presents two phases:

(a) a quiet phase (1978-84), characterized by a mean radius $r / r_{*}=5.2$;

(b) the beginning of an expanding phase (1984-87), where the radius increases to $r / r_{*}=8.6$.

From 1987 to 1998 , the radius of the $\mathrm{CE}$ deduced from $\mathrm{H} \beta$ and $\mathrm{Fe}$ II $\lambda 4924 \AA$ emission lines show a regular increase; the radius deduced from the $\mathrm{H} \beta$ line increased by a factor of 2.4 in 11 years, while the radius deduced from the Fe II $\lambda 4924 \AA$ line increased by a factor 2 in 4 years. In 1996 the largest outer radius, $r / r_{*}=25.0$, is attained. This radius has begun to decrease since then and this tendency must be confirmed by new observations.

The present emission phase of Pleione can be compared with the previous one, which elapsed from 1954 to 1972 . Spectra dating back to 1960 (unpublished) show that the $\mathrm{H} \beta$ line profiles are similar in shape and have similar emission peak separations and RVs than the 1987-98 ones. This means that possibly the present outer radius must decrease in the following years if Pleione has to enter a new Be-shell phase similar to that of 1972 .

These observations show that Be phase changes are characterized by a huge variation of the $\mathrm{CE}$ extent and that CEs producing Be phases are larger than those producing Be-shell phases. These may also imply that changes of density and temperature distribution in CEs should play a more important role for $\mathrm{Be}$ phase transition than their geometry.

\section{References}

Ballereau, D., Chauville, J., Mekkas, A. 1988, A\&ASS 75, 139

Huang, S.-S. 1972, ApJ 171, 549

Katahira, J., Hirata, R., Ito, M., Katoh, M., Ballereau, D., Chauville, J. 1996, PASJ 48, 317 